Accepted Version

The final authenticated version is available online at https://doi.org/10.1007/978-3-030-22744-9_43.

\title{
Surrogate-based optimization of tidal turbine arrays: A case study for the Faro-Olhão Inlet
}

\author{
Eduardo González-Gorbeña ${ }^{1[0000-0003-1996-7820]}$, André Pacheco ${ }^{1[0000-0002-8340-0629]}$, \\ Theocharis A. Plomaritis ${ }^{20000-0002-9819-0927]}$, Óscar Ferreira ${ }^{1}$, Cláudia Sequeira ${ }^{1}$, and \\ Theo Moura ${ }^{10000-0003-2322-7518]}$. \\ ${ }^{1}$ Centre for Marine and Environmental Research, Universidade do Algarve \\ Ed. 7, Campus de Gambelas, 8005-139, Faro, Portugal. \\ \{egeisenmann, ampacheco, oferreir, cdsequeira, \\ tgmoura\} eualg.pt \\ http://www.cima.ualg.pt/ \\ ${ }^{2}$ Faculty of Marine and Environmental Science, Department of Earth Science, Universidad de \\ Cádiz, Campus Rio San Pedro (CASEM), Puerto Real 11510, Cádiz, Spain. \\ haris.plomaritis@uca.es
}

\begin{abstract}
This paper presents a study for estimating the size of a tidal turbine array for the Faro-Olhão Inlet (Potugal) using a surrogate optimization approach. The method compromises problem formulation, hydro-morphodynamic modelling, surrogate construction and validation, and constraint optimization. A total of 26 surrogates were built using linear RBFs as a function of two design variables: number of rows in the array and Tidal Energy Converters (TECs) per row. Surrogates describe array performance and environmental effects associated with hydrodynamic and morphological aspects of the multi inlet lagoon. After validation, surrogate models were used to formulate a constraint optimization model. Results evidence that the largest array size that satisfies performance and environmental constraints is made of 3 rows and 10 TECs per row.
\end{abstract}

Keywords: Hydro-morphodynamic modelling, Marine renewable energy, Ria Formosa.

\section{Introduction}

The sustainable development of island or isolated communities is getting in the agenda of governments all around the world. In Europe, the European Commission is promoting initiatives to help islands generate their own sustainable, low-cost energy [1]. Tidal current energy is a form of marine renewable energy, which converts the kinetic energy of the tides into electricity using Tidal Energy Converters (TECs). As tides are perfectly predictable and have a significant energy density, tidal current energy becomes a reliable source of clean energy if compared with other sources of renewable energy, such as waves or wind. The main drawback is that it is very site specific, i.e. there are not too many places around the world with feasible conditions for commercial exploration. As with wind energy, in order to decrease the levelized cost of electricity (LCOE) tidal 
turbines need to be grouped in arrays. Reasons that served to conduct research in TEC arrays optimization.

Initially, optimization strategies that were developed to answer the TEC array problem have been inspired by the wind energy industry, whose primary objective is to maximize power output by reducing wake interferences between turbines within the array [2]. For this purpose, analytical wake models were developed to model these effects, being the Jensen wake model [3] one of the most popular methods. The main difference between the effects on flow due to wind and tidal turbines is that the former occupies a little portion of the vertical profile of the flow, while the latter, usually, occupies more than $1 / 3$ of the flow depth. This causes that the flow is not only affected downstream the turbines but also upstream and around the turbines. Flow effects are more significant when increasing the number of turbines, which can be felt far away from the array deployment and can result in environmental impacts. It is for this reason that simple analytical models like those used in [2] are not suitable for tidal array arrays, especially when turbines are placed in complex environments. Therefore, any proposed tidal energy array design method has to be fully coupled with the flow to ensure a proper optimization, to take the most of the resource while trying to reduce as much as possible detrimental environmental impacts.

In the literature, there are two approaches that tackle this problem. Both approaches are fully coupled by using numerical models that solve the shallow water equations. In the first approach, developed by Funke et al. [4], the gradient of the array power output is computed using the adjoint technique of variational calculus. The advantage of this approach is that the computational cost is independent of the number of turbines that compose the array and allows the free position of each de-vice within the domain defined. The main drawbacks of this approach are that it requires the development of an adjoint solver, which might be difficult depending on the software being used to solve the hydrodynamics, and that the iterative process yields an optimum solution not giving the possibility to explore the design variable domain by changing the constraint values without repeating the calculation. The inclusion of environmental constraints is still under development. For the moment, environmental impacts are only considered in terms of the effects on flow velocities at specific regions [5].

On the other hand, in the approach of González-Gorbeña et al. [6], the optimum tidal array is searched by means of surrogates based on a set of expensive computer experiments, a method known as surrogate-based optimization [7]. The SBO method consists in fitting a mathematical function to approximate a more time-consuming function. The main advantages are that once a surrogate is validated, the whole de-sign variable space can be explored instantaneously, thus giving the possibility to assess efficiently the effect of changing the constraints limits on the objective function. Moreover, it can be implemented in any set of data, independently of the soft-ware in which the responses are generated, and surrogates can represent any environmental impact that the simulations can generate, e.g. shear velocities [8], tide discharges and morphological changes [9]. The main disadvantage of the SBO approach is that the number of simulations that are necessary to build a surrogate is a function of the number of design variable defined in the problem. If the free positioning of turbines is considered, this approach is 
impractical for the optimization of large arrays, as the position of each device is defined by at least a pair of design variables (i.e. the $\mathrm{x}$ and $\mathrm{y}$ horizontal coordinates).

In this paper, it is presented a case study applying the SBO approach where it is estimated the maximum size of a tidal array for the Faro-Olhão Inlet considering performance and environmental constraints. The paper has the following organization: Section 2 describes the case study region; Section 3 details the SBO approach adopted; Section 4 presents the results of the design space exploration and optimization models; finally, Section 5 provides a discussion of the results and the conclusion of the study.

\section{Site description}

The Ria Formosa is coastal lagoon system with multiple inlets placed in Southern Portugal (Fig. 1). The system has two peninsulas and five islands enclosing an area with, sand flats, salt marshes and a complex system of tidal channels. Small communities of fishermen live on these islands. There are six inlets that connect the lagoon with the ocean; two of them are stabilized with jetties at both sides (Faro-Olhão and Tavira inlets) and the rest are free to migrate (Ancão, Armona, Fuseta and Lacém). The FaroOlhão Inlet and the Armona Inlet together represent almost $90 \%$ of the total tidal prism of the lagoon. During spring and neap tides the Faro-Olhão Inlet provides $61 \%$ and $45 \%$, respectively, of the overall tidal prism, while the Armona Inlet accounts $23 \%$ and $40 \%$, correspondingly [10]. The dynamics that force these discharges are due to the semi-diurnal tides that the region experiences, with average elevation ranges of $2.8 \mathrm{~m}$ for spring tides and $1.3 \mathrm{~m}$ for neap tides. Both inlets have an ebb dominated behavior (i.e. shorter ebb duration generate higher mean ebb velocity). However, at the FaroOlhão Inlet, the flood prism is considerably greater than the ebb prism, therefore sediment deposition occurs inside the lagoon, while for the Armona Inlet sediment flushes seaward. As a result, due to the importance of the Faro-Olhão Inlet, any alteration of the inlet could disrupt the dynamic equilibrium of the whole system which can result in adverse environmental impacts.

\section{Surrogate-based optimisation approach}

Surrogate-based optimization has been applied in various branches of knowledge, including aeronautical [11], automotive [12], and telecommunications [13], among others. As previously said, it is a very popular method when time consuming computer simulations are involved in the design process. The SBO approach compromises problem formulation, design of experiments, computer simulations, surrogate construction and validation, and mathematical optimization. Fig. 2 summarizes the SBO approach flowchart and the following subsections detail each of the steps adopted to estimate the maximum capacity of the Faro-Olhão Inlet for tidal energy extraction. 


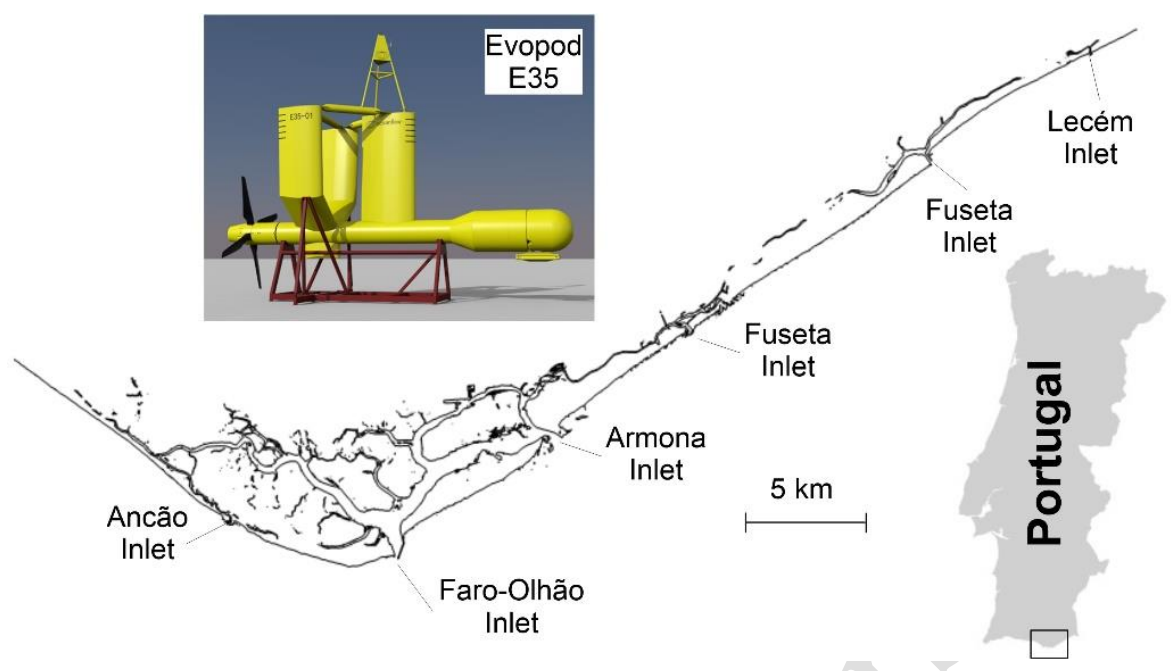

Fig. 1. Location map of the region of study and 3D model of Evopod ${ }^{\mathrm{TM}} 35 \mathrm{~kW}$ (E35) tidal energy converter.

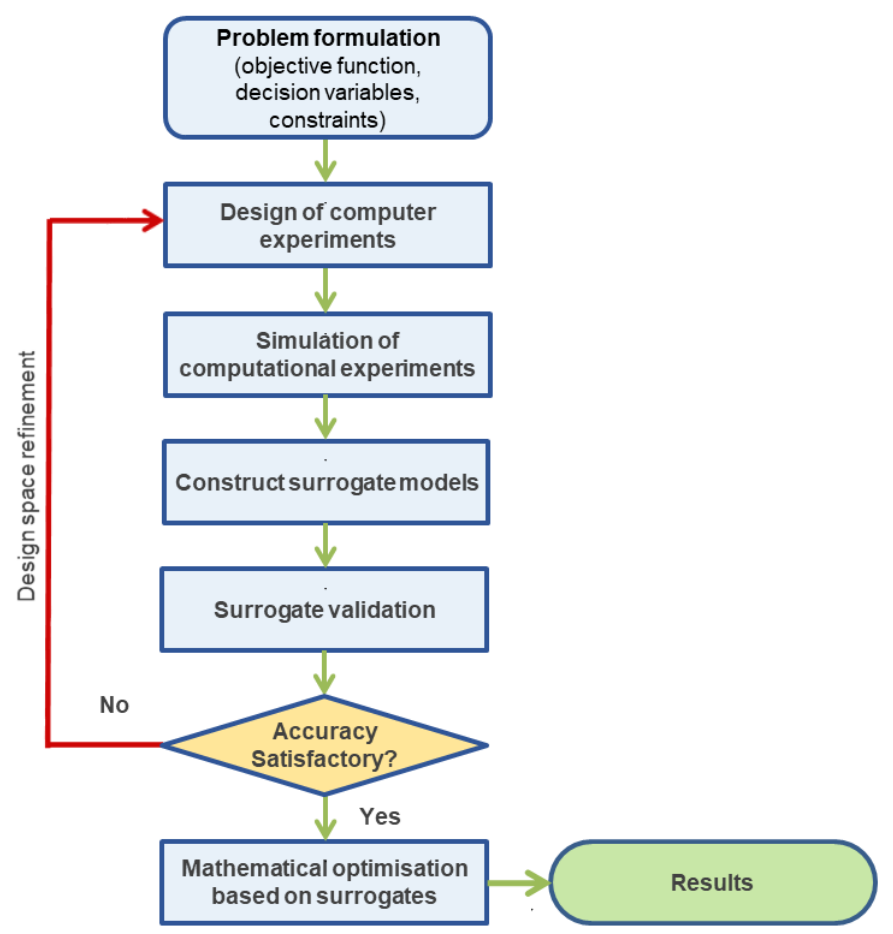

Fig. 2. Surrogate based optimization methodology flowchart. 


\section{Problem formulation}

The first step in the SBO approach is to define what are the dependent and independent variables that describe the problem to be solved. In order to decrease as much as possible the number of design variables, these need to be carefully selected. Given a site with potential for a tidal stream development, arrays can be defined in terms of the individual position of each of the turbines that compose the farm or in terms of the TECs per row and number of rows that form the array. The former approach implies that each turbine should be defined in terms of its coordinates, which implies that the number of design variable by a factor of 3 (e.g. x-y-z coordinates) or 2 (e.g. x-y coordinates) for each TEC within the array. As mentioned above, this will lead to a computationally unaffordable approach when considering a large array made of hundreds of devices. Instead, given a predefined number of rows and TECs per row, arrays can be denoted as a function of two design variables, these are the longitudinal spacing between rows and the lateral spacing among devices within a row [5]. This approach entails that arrays should have a uniform distribution inline (i.e. downstream TECs have $0^{\circ}$ phase difference respect upstream devices) or staggered (i.e. downstream TECs have $90^{\circ}$ phase difference respect upstream devices). In order to overcome this problem, TEC arrays can be defined in terms of four design variables, these are: the longitudinal, lateral, vertical and staggered spacings [8]. The approaches of [5] and [8] imply the use of continuous variables, which entails the re-meshing of the domain for each computer simulation. This represents a drawback when modelling environmental hydraulics of complex lagoons where domain meshing is a laborious task and TECs are modelled in a sub-grid scale (i.e. the length scale of the computational grid is larger than the length scale of the TEC). In this study, it is adopted the strategy presented in [9], where a tidal array was defined in terms of two discrete design variables, the number of rows and the number of TECs per row. The Evopod ${ }^{\mathrm{TM}} 35 \mathrm{~kW}$ TEC (Fig. 1) was selected to form the array. The limits of each of the design variables were defined considering model grid discretization, hydrodynamic conditions, turbine operation specifications and geometric constraints i.e. depth and width of the Faro-Olhão Channel. As it can be observed from Fig. 3 , the largest resource is at the inlet throat, where occurrences of tidal current velocities greater than $0.7 \mathrm{~m} / \mathrm{s}$ are larger than $60 \%$ of the time. It was here that was positioned the first row of turbines with a minimum and maximum number of 6 TECs and 11 TECs, respectively. This implies that the minimum and maximum lateral spacing is 3 (to avoid turbine collision) and 6 rotor diameters (blockage effects are negligible with larger spacings), correspondingly. On the other hand, considering a uniform longitudinal spacing of 20 rotor diameters (to ensure wake recovery) and a minimum threshold of $25 \%$ of the time with occurrences of flow velocities greater than the cut-in velocity, the maximum number of array rows was set to 13. Fig. 3 displays the deposition of the TEC rows for a hypothetical array of 13 rows.

Regarding the dependent variables, array performance is usually measured in terms of its Capacity Factor, $C F$, which denotes the percent of time that the array is operating at rated power. Therefore, the performance of the array can be described by the overall array efficiency as well as by the average of the efficiencies of array rows. Assess and quantify the effects of TEC arrays on the environment is a more difficult task, 
principally in what regards to identify what are the adverse impacts and, consequently, to define the thresholds of what is considered a negative impact or not. Therefore, environmental impacts will be project-specific. In this particular study, a set of environmental impacts related with hydrodynamic and morphological effects was defined as function of the above mentioned design variables.

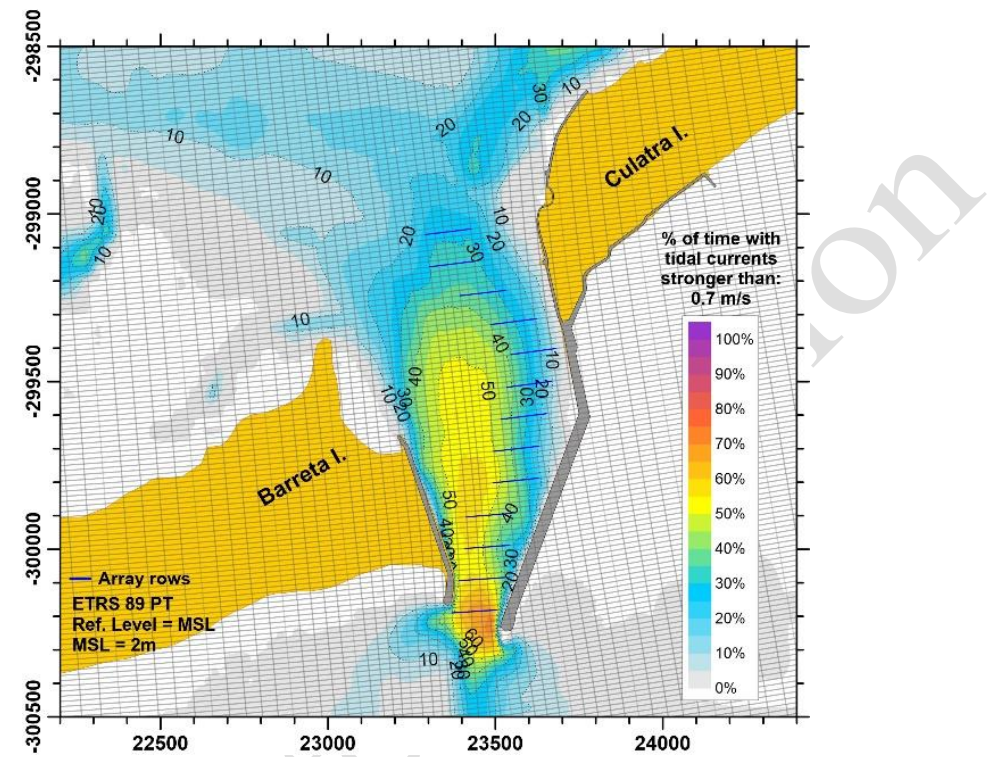

Fig. 3. Contour map showing percent of time with tidal currents above $0.7 \mathrm{~m} / \mathrm{s}$.

\subsection{Design of computer experiments}

A design of experiments (DoE) consists in generating a set of observation points relating the independent variables to adequately describe the design space. The objective of the DoE is to minimize as much as possible the error between the real-function (i.e. the computer simulation) and the fitted function the (i.e. the surrogate) using the least number of observations. In the literature, there is a lot of research to attain this goal, where new methods are proposed frequently. For a classical review on this matter see ref. [14]. DoEs can have a pre-defined number of sample plans or can follow an adaptive-sequential approach. There are no rules to define the minimum number of sample points but, generally, is not less than times the number of design variable [15]. Following the conclusions of [16], in this study, a fixed sample plan size with 15 points per variable was chosen to characterize the design space plus 3 additional points to validate the surrogates. Sample points were selected manually and Fig. 4 shows the initial design plan. 


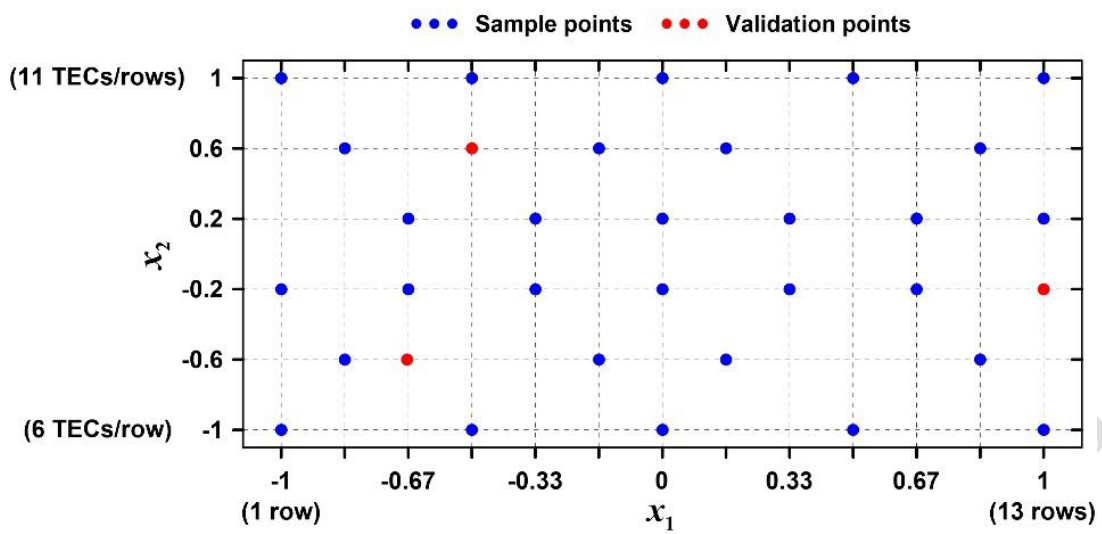

Fig. 4. Initial design of experiments sample plan (blue points) and validation points (red points).

\subsection{Computational simulations}

After the sample plan was defined, the computer experiments were executed using the modelling package Delft3D [17] in its two-dimensional depth averaged version (2DH). Full details about the set-up, TEC parameterization, calibration and validation of the hydro-morphodynamic model of the entire Ria Formosa are available in ref [9].

A total of 26 responses were obtained for each of the 33 simulations compromising performance and environmental indicators. The performance indicators are: the overall array capacity factor, $C F_{\text {Array }}$; and average capacity factor of each array row, $C F_{i}$. The environmental indicators are: (i) percent variations of cumulative flood and ebb instantaneous discharges $\left(\Delta \Sigma Q_{i}\right)$ throughout a spring tide cycle at Faro-Olhão and Armona tidal inlets and for the whole lagoon system; (ii) percent changes of the cumulative flood and ebb instantaneous discharges ratio between Armona and the Faro-Olhão inlets $\left(\Delta \Sigma Q_{A r} / \Delta \Sigma Q_{F O}\right)$; and (iii) net sediment volume differences $(\Delta V)$ and variations in average depth changes $\left(\Delta h_{\text {avg }}\right)$ for the Armona Inlet and the Faro-Olhão Inlet flood delta.

In order to assess the array and its rows performance as well as the array impacts on hydrodynamics and morphodynamics of Ria Formosa natural park, a set of threshold limits have been defined and summarised in Table $\mathbf{1}$ to Table $\mathbf{3}$.

Table 1. Constraints values for capacity factors of the entire array, $C F_{\text {Array }}$, and for each row of the array, $C F_{i}$.

\begin{tabular}{lcccccccccccccc}
\hline Constr. No & $\mathbf{1}$ & $\mathbf{2}$ & $\mathbf{3}$ & $\mathbf{4}$ & $\mathbf{5}$ & $\mathbf{6}$ & $\mathbf{7}$ & $\mathbf{8}$ & $\mathbf{9}$ & 10 & 11 & 12 & 13 & 14 \\
\hline \multirow{2}{*}{ Constraint } & $\mathrm{CF}_{\text {Array }}$ & $\mathrm{CF}_{1}$ & $\mathrm{CF}_{2}$ & $\mathrm{CF}_{3}$ & $\mathrm{CF}_{4}$ & $\mathrm{CF}_{5}$ & $\mathrm{CF}_{6}$ & $\mathrm{CF}_{7}$ & $\mathrm{CF}_{8}$ & $\mathrm{CF}_{9}$ & $\mathrm{CF}_{10}$ & $\mathrm{CF}_{11}$ & $\mathrm{CF}_{12}$ & $\mathrm{CF}_{13}$ \\
& {$[\%]$} & {$[\%]$} & {$[\%]$} & {$[\%]$} & {$[\%]$} & {$[\%]$} & {$[\%]$} & {$[\%]$} & {$[\%]$} & {$[\%]$} & {$[\%]$} & {$[\%]$} & {$[\%]$} & {$[\%]$} \\
\hline Value & 12.5 & 15 & 12 & 10 & 10 & 10 & 10 & 10 & 10 & 10 & 10 & 10 & 10 & 10 \\
\hline
\end{tabular}


Table 2. Environmental constraints values for flood and ebb spring discharges $(\Delta \Sigma Q)$ of Armona $\left({ }_{A r}\right)$ and Faro-Olhão $(F O)$ inlets, together with their discharges fraction $\left(\Sigma Q_{A r} / \Sigma Q_{F O}\right)$. Subscripts $e$ and $f$ depict for ebb and flood tides, respectively.

\begin{tabular}{|c|c|c|c|c|c|c|c|c|}
\hline Constr. № & 15 & 16 & 17 & 18 & 19 & 20 & 21 & 22 \\
\hline Constr. & $\begin{array}{c}\Delta \Sigma Q_{f, F o} \\
{[\%]}\end{array}$ & $\begin{array}{c}\Delta \Sigma Q_{f, A r} \\
{[\%]}\end{array}$ & $\begin{array}{c}\Sigma Q_{f, A r} / \Sigma Q_{f, F o} \\
{[\%]}\end{array}$ & $\begin{array}{c}\Delta \Sigma Q_{f, \text { all }} \\
{[\%]}\end{array}$ & $\begin{array}{c}\Delta \Sigma Q_{e, F O} \\
{[\%]}\end{array}$ & $\begin{array}{c}\Delta \Sigma Q_{e, A r} \\
{[\%]}\end{array}$ & $\begin{array}{c}\Sigma Q_{e, A r} / \Sigma Q_{e} \\
\text { Fo }[\%]\end{array}$ & $\begin{array}{c}\Delta \Sigma Q_{e, a l l} \\
{[\%]}\end{array}$ \\
\hline Value & -10 & 10 & 7.5 & -5 & -5 & 2.5 & 2.5 & -2.5 \\
\hline
\end{tabular}

Table 3. Environmental constraints values for morphological aspects. $\Delta h_{\text {avg }}$ and $\Delta V_{\text {net }}$ depict the average depth and net sediment volume variations, respectively, for Armona $(\mathrm{Ar})$ and FaroOlhão $(\mathrm{FO})$ inlets.

\begin{tabular}{lcccc}
\hline Constr. No & $\mathbf{2 3}$ & $\mathbf{2 4}$ & $\mathbf{2 5}$ & $\mathbf{2 6}$ \\
\hline \multirow{2}{*}{ Constraint } & $\begin{array}{l}\Delta \boldsymbol{V}_{\text {net, Fo }} \\
{\left[\mathrm{m}^{3} / \mathrm{yr}\right]}\end{array}$ & $\begin{array}{l}\Delta \boldsymbol{V}_{\text {net, } \text { Ar }} \\
{\left[\mathrm{m}^{3} / \mathrm{yr}\right]}\end{array}$ & $\begin{array}{c}\Delta \boldsymbol{h}_{\text {avg, Fo }} \\
{[\mathrm{cm} / \mathrm{yr}]}\end{array}$ & $\begin{array}{c}\Delta \boldsymbol{h}_{\text {avg, Ar }} \\
{[\mathrm{cm} / \mathrm{yr}]}\end{array}$ \\
\hline Value & $-10,000$ & 40,000 & -5 & 15 \\
\hline
\end{tabular}

\subsection{Surrogate construction and validation}

The data generated though the computational simulations was used to train a set of surrogates that approximate the entire design variable space. In the literature, there are many candidates to use as surrogates For a review on surrogates, readers can refer to [18]. In the present study, linear Radial Basis Functions (RBFs) were used to build the surrogates.

For exact data interpolation in a multi-dimensional space, $\mathrm{RBF}$ is a popular technique [19]. A response, $y$, is related to a vector of input variables, $\mathbf{x}$, through a linear combination of the basis functions. As a real valued function, RBF data points, $\mathbf{x}$, affect their distance, $r$, from another data point, $\mathbf{x}_{i}$, named a center. The Euclidian norm, $\mathrm{r}=$ $\left\|\mathbf{x}-\mathbf{x}_{i}\right\|$ norm represents the distance between the two points. Therefore, a data point in a data set will affect to a greater extent the nearer points than the faraway points, in such a way that $\phi\left(\mathbf{x}, \mathbf{x}_{i}\right)=\phi(r)=\phi\left(\mathbf{x}-\mathbf{x}_{i}\right)$. In this manner, the way data points are related depends on the basis function selected. Some of the most common basis functions are: linear, cubic, Gaussian, thin-plate-spline, inverse multiquadric, and multiquadric.

Consequently, it is possible to construct the approximation response function, $\hat{y}_{i}$, using the following expression:

$$
\hat{y}_{j}=\mathbf{w}^{\mathbf{T}} \phi=\sum_{i, j=1}^{n} w_{i} \phi\left(\left\|\mathbf{x}_{j}-\mathbf{x}_{i}\right\|\right), \quad i, j=1,2, \ldots, n
$$

here, $\mathbf{w}$ is a vector comprising the weights, $w_{i}$, for the linear arrangement of basis vectors that are in the vector $\phi$; which provides the expression,

$$
y=w^{T} \Phi
$$


where y represents the vector with the results from the computer model and $\Phi$ is the matrix containing the combination of linear basis vectors.

Finally, weights values can be obtained employing the least squares estimator given by Eq. (3), i.e.

$$
\hat{\mathbf{w}}=\left(\boldsymbol{\Phi}^{\mathrm{T}} \boldsymbol{\Phi}\right)^{-1} \boldsymbol{\Phi}^{\mathrm{T}} \mathbf{Y}
$$

Once the surrogates were built, the prediction capabilities of each of the surrogates were assessed using a leave- $k$-out $(k=3)$ cross validation technique [20].

The Normalised Root Mean Square Error (NRMSE) and the Normalised Maximum Error (NMAXE) were used to assess the predictive capabilities of the surrogates. Forrester et al. [20] suggests that values of a NRMSE $<0.1$ and NRMSE $<0.02$ imply surrogates with reasonable and exceptional predictive abilities, respectively. In this study, NRMSE and NMAXE values for all surrogates were below 0.01 .

\subsection{Constraint optimisation}

After validation, surrogate models were used to formulate a multi-objective constrained optimisation model that maximises the number of tidal turbines and associated power output for the Faro-Olhão Inlet subject to performance and environmental restrictions. The mathematical model is given by:

$$
\text { Maximise }\left\{x_{1} x_{2}, P_{\text {Array }}\right\}
$$

Subject to:

$$
\begin{aligned}
& P_{\text {Array }}=C F_{\text {Array }}(\mathbf{x}) x_{1} x_{2} P_{r} t \\
& \hat{g}_{i}(\mathbf{x}) \geq b_{i}, \quad \forall i \in\left\{1, \ldots, x_{2}+1\right\} \\
& \hat{g}_{i}(\mathbf{x}) \geq b_{i}, \quad \forall i \in\{15,18,19,22,23,25\} \\
& \hat{g}_{i}(\mathbf{x}) \geq b_{i}, \quad \forall i \in\{16,17,20,21,24,26\} \\
& x_{1} \in\{1, \ldots, 13\}, x_{2} \in\{6, \ldots, 11\}
\end{aligned}
$$

Equation (4) defines the objective functions to be maximized. Equation (5) defines how to calculate the overall power output of the array, where $P_{r}$ depicts the turbine's rated $(35 \mathrm{~kW})$, and the time interval to calculate power production (364 days.yr-1). Equation (6) defines the set of constraints related with array and row efficiency (Table 1). Equations (7), and (8) represent the set of environmental constraints, i.e. the values of the constraints in Table 2 and Table 3. Finally, equation (9) declares the values of the design variables, i.e. the number of array rows and TECs per row. Notice that the integer value of the design variable representing the number of array row defines the rows that are active. The first row of the array is placed at the inlet throat, then subsequent rows are placed consecutively toward the interior of the lagoon. 


\section{Design space exploration and optimisation results}

In order to understand how sensitive the responses are to the design variables, the domain space of each of the surrogates were explored for all the possible combinations of the discrete values of the design variables, $x_{1}=[1, \ldots, 13]$ and $x_{2}=[6, \ldots, 11]$. Fig. 5 to Fig. 7 summarizes graphically the results obtained.
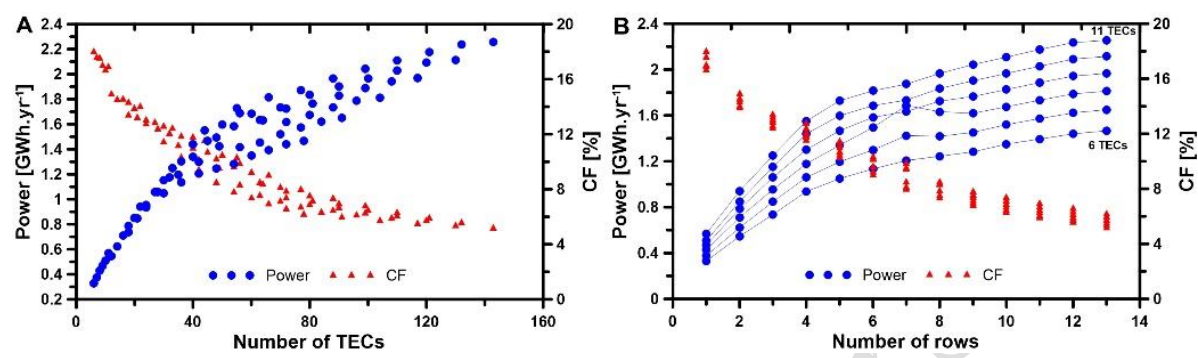

Fig. 5. Array capacity factor and annual power output related with A) quantity of TECs, and B) quantity of rows.
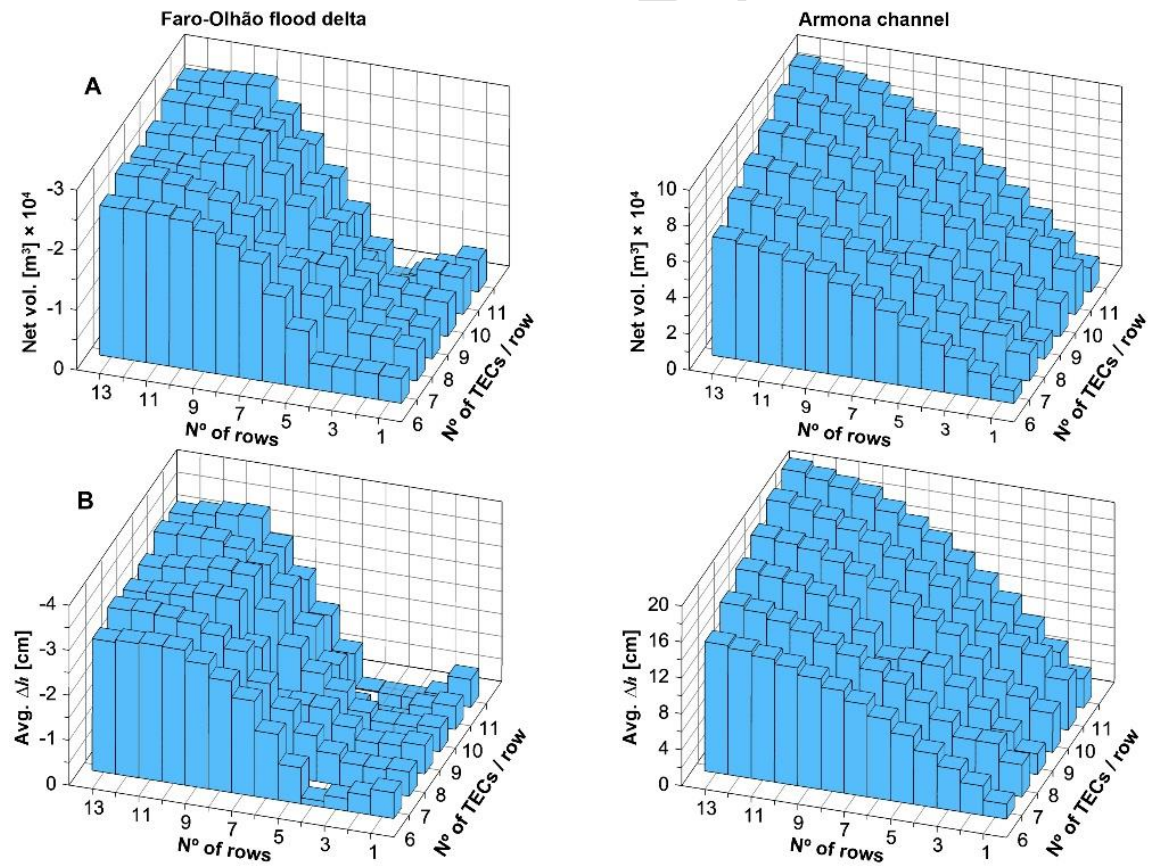

Fig. 6. Bar charts illustrating: A) sedimentation and erosion net volume changes and B) average depth variations for the Faro-Olhão flood delta (left) and Armona inlet (right). Each of the bars represent one of the possible solutions (i.e. 78 array layouts. Negative and positive values indicate decrease and increase of scalar quantities, respectively. Notice that the Armona Inlet experiences erosion and the Faro-Olhão flood delta suffers sedimentation. 

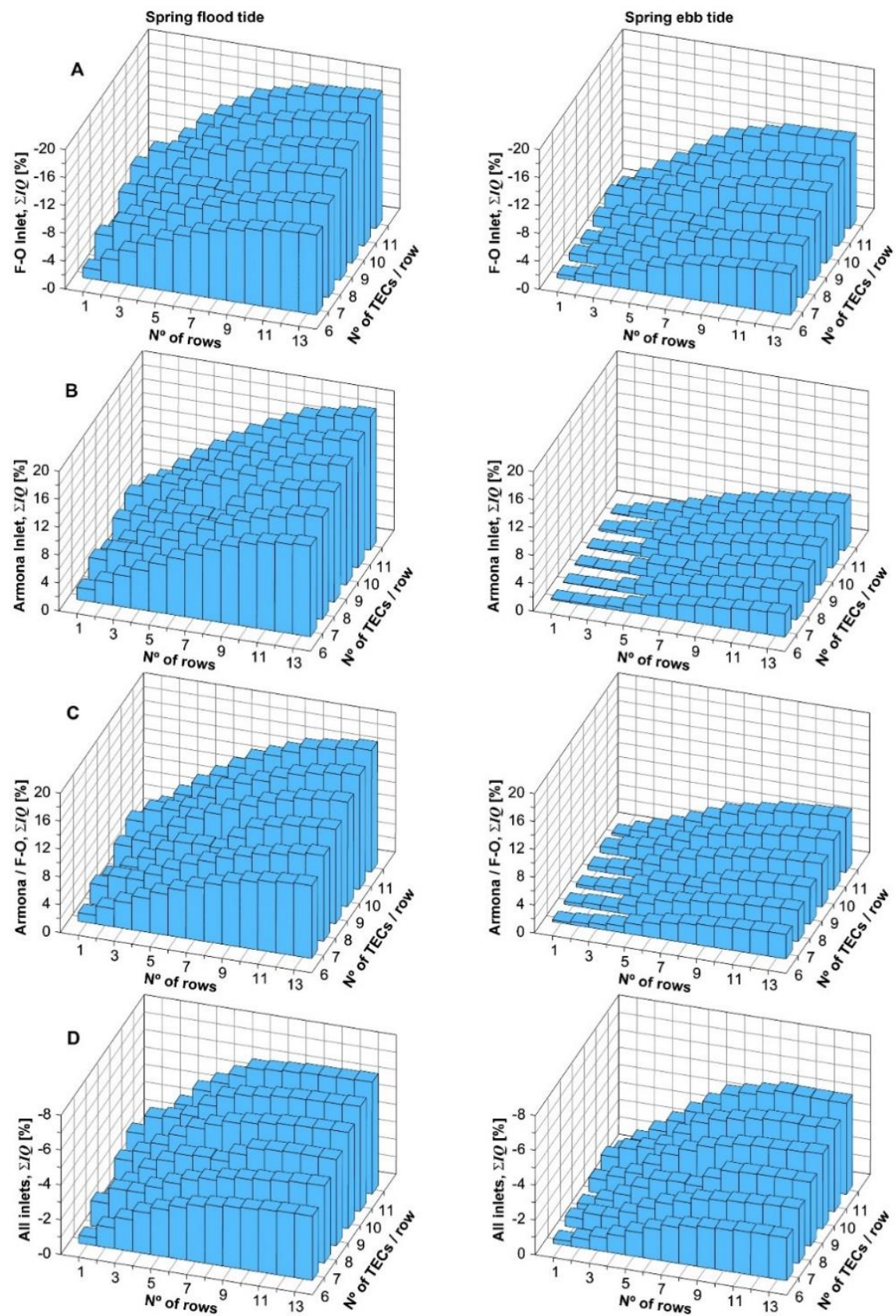

Fig. 7. Bar charts illustrating: (A and $B$ ) changes of immediate cumulative discharges and (C) discharge fractions for the Armona and Faro-Olhão, and (D) variations of immediate cumulative discharges for all inlets during flood (left) and ebb (right). Each of the bars represent one of the possible solutions (i.e. 78 array layouts. The baseline fraction between Armona and FaroOlhão inlets for ebb and flood discharges are $35.8 \%$ and $37.8 \%$, respectively. 
By exploring the design variable domain, it was possible to conclude that: 1) the most productive arrays were those with smaller number of rows; 2) the number of TECs per row does not affect the capacity factor as much as the number of array rows does; 3 ) once a certain quantity of TECs in an array is achieved, the placement of additional turbines does not contribute significantly to increase the overall power production; 4) the magnitude of the environmental responses were proportional to the quantity of TECs within the array, except for the morphological changes of the Faro-Olhão flood delta, which were more influenced by the number of rows than by the number of TECs.

Table 4. Values of the design variables, $x_{1}$ and $x_{2}$, array capacity factor, $C F_{\text {Array }}$, and power output, $P_{\text {Array }}$, for the optimal solution, $x^{*}$.

\begin{tabular}{ccccc}
\hline$x_{1}[$ rows] & $x_{2}[\mathrm{TECs} /$ row] & $\boldsymbol{x}^{*}[\mathrm{TECs}]$ & $\boldsymbol{C F}_{\text {Array }}[\%]$ & $\boldsymbol{P}_{\text {Array }}\left[\mathrm{GWh} . \mathrm{yr}^{-1}\right]$ \\
\hline 3 & 10 & 30 & 12.6 & 1.20 \\
\hline
\end{tabular}

Under the constraints imposed, the results from the optimisation models revealed that arrays with 30 TECs distributed in 3 rows with 10 devices each were the maximum allowable array size for the Faro-Olhão Inlet satisfying the performance constraints (Table 5) and without adversely affecting the environment (Table 6 and Table 7).

Table 5. Constraints values model results for capacity factors of the entire array, $C F_{\text {Array }}$, and for each row of the array, $C F_{i}$.

\begin{tabular}{lcccccccccccccc}
\hline Constr. № & $\mathbf{1}$ & $\mathbf{2}$ & $\mathbf{3}$ & $\mathbf{4}$ & $\mathbf{5}$ & $\mathbf{6}$ & $\mathbf{7}$ & $\mathbf{8}$ & $\mathbf{9}$ & 10 & 11 & 12 & 13 & 14 \\
\hline \multirow{2}{*}{ Constr. } & $\mathrm{CF}_{\text {Array }}$ & $\mathrm{CF}_{1}$ & $\mathrm{CF}_{2}$ & $\mathrm{CF}_{3}$ & $\mathrm{CF}_{4}$ & $\mathrm{CF}_{5}$ & $\mathrm{CF}_{6}$ & $\mathrm{CF}_{7}$ & $\mathrm{CF}_{8}$ & $\mathrm{CF}_{9}$ & $\mathrm{CF}_{10}$ & $\mathrm{CF}_{11}$ & $\mathrm{CF}_{12}$ & $\mathrm{CF}_{13}$ \\
& {$[\%]$} & {$[\%]$} & {$[\%]$} & {$[\%]$} & {$[\%]$} & {$[\%]$} & {$[\%]$} & {$[\%]$} & {$[\%]$} & {$[\%]$} & {$[\%]$} & {$[\%]$} & {$[\%]$} & {$[\%]$} \\
\hline Value & 12.6 & 15.3 & 12.2 & 10.1 & -- & --- & -- & -- & -- & -- & -- & -- & --- & -- \\
\hline
\end{tabular}

Table 6. Models results of the environmental constraints for flood and ebb spring discharges $(\Delta \Sigma Q)$ of Armona $(A r)$ and Faro-Olhão $(F O)$ inlets, together with their discharges fraction $\left(\Sigma Q_{A r} / \Sigma Q_{F O}\right)$. Subscripts $e$ and $f$ depict for ebb and flood tides, respectively.

\begin{tabular}{|c|c|c|c|c|c|c|c|c|}
\hline Constr. № & 15 & 16 & 17 & 18 & 19 & 20 & 21 & 22 \\
\hline Constraint & $\begin{array}{c}\Delta \Sigma Q_{f, F o} \\
{[\%]}\end{array}$ & $\begin{array}{c}\Delta \Sigma Q_{f, A r} \\
{[\%]}\end{array}$ & $\begin{array}{c}\Sigma Q_{f, A r} / \Sigma Q_{f, F O} \\
{[\%]}\end{array}$ & $\begin{array}{c}\Delta \Sigma Q_{f, \text { all }} \\
{[\%]}\end{array}$ & $\begin{array}{c}\Delta \Sigma Q_{e, F O} \\
{[\%]}\end{array}$ & $\begin{array}{c}\Delta \Sigma Q_{e, A r} \\
{[\%]}\end{array}$ & $\begin{array}{c}\sum Q_{e, A r} / \Sigma Q_{e} \\
\quad F O[\%]\end{array}$ & $\begin{array}{c}\Delta \Sigma Q_{e, a l l} \\
{[\%]}\end{array}$ \\
\hline Value & -9.4 & 7.8 & 7.1 & -3.7 & -4.0 & 1.0 & 1.9 & -1.9 \\
\hline
\end{tabular}

Table 7. Model results of the environmental constraints for morphological aspects. $\Delta h_{\text {avg }}$ and $\Delta V_{\text {net }}$ depict the average depth and net sediment volume variations, respectively, for Armona $(A r)$ and Faro-Olhão $(F O)$ inlets.

\begin{tabular}{lcccc}
\hline Constraint No & $\mathbf{2 3}$ & $\mathbf{2 4}$ & $\mathbf{2 5}$ & $\mathbf{2 6}$ \\
\hline \multirow{2}{*}{ Constraint } & $\begin{array}{l}\Delta \boldsymbol{V}_{\text {net, Fo }} \\
{\left[\mathrm{m}^{3} / \mathrm{yr}\right]}\end{array}$ & $\begin{array}{c}\Delta V_{\text {net, } \text { Ar }} \\
{\left[\mathrm{m}^{3} / \mathrm{yr}\right]}\end{array}$ & $\begin{array}{c}\Delta h_{\text {avg, Fo }} \\
{[\mathrm{cm} / \mathrm{yr}]}\end{array}$ & $\begin{array}{c}\Delta h_{\text {avg, Ar }} \\
{[\mathrm{cm} / \mathrm{yr}]}\end{array}$ \\
\hline Value & -3013 & 37019 & 0.1 & 8.4 \\
\hline
\end{tabular}




\section{Conclusions}

In this study, the maximum permissible capacity of the Faro-Olhão Inlet for tidal current energy exploration was estimated by means of using a surrogate-based optimisation. Tidal arrays were defined as a function of a pair of design variables, these are: 1) number of TEC rows and 2) number of TECs per array row. A 2DH hydro-morphodynamic model was used to compute a set of scenarios to assess performance and environmental effects of tidal arrays in Ria Formosa. Linear RBF were selected to build surrogates from the responses of the numerical simulations. Finally, employing validated surrogates, a multi-objective optimisation model was formulated to maximize array quantity of TECs and power output, while minimizing detrimental environmental impacts. Results suggest that the optimum solution consist of an array with 10 TECs per row and a total of 3 rows.

The main advantages of using the surrogate-based approach for optimizing tidal energy arrays can be summarized as follows:

- The SBO approach results very useful when the use expensive computational simulations is involved;

- High complex response that are challenging to model with simple analytical functions can be approximated by using surrogates based on high definition numerical models;

- Both the objective function and the constraints can be represented by surrogates;

- Allows to formulate constraint optimisation models;

- The optimisation model can incorporate environmental and performance constraints; and

- In multidimensional problems, the SBO approach allows to search efficiently (i.e. using less computational resources) the whole design space.

\section{Acknowledgements}

Eduardo González-Gorbeña has received funding for the OpTiCA project (http://mscaoptica.eu/) from the Marie Skłodowska-Curie Actions of the European Union's H2020MSCA-IF-EF-RI-2016 / GA\#: 748747. The paper is a contribution to the SCORE project, funded by the Portuguese Foundation for Science and Technology (FCTPTDC/AAG-TEC/1710/2014). André Pacheco was supported by the Portuguese Foundation for Science and Technology under the Portuguese Researchers' Programme 2014 entitled "Exploring new concepts for extracting energy from tides" (IF/00286/2014/CP1234).

\section{References}

1. European Commission: Factsheet: Clean Energy for Islands Initiative. Available online at: https://ec.europa.eu/commission/sites/beta-political/files/clean-energy-islands-initiative_en.pdf 
2. Brutto, O. A. L., Thiébot, J., Guillou, S. S., Gualous, H. A semi-analytic method to optimize tidal farm layouts-Application to the Alderney Race (Raz Blanchard), France. Applied energy 183 (2016) 1168-1180.

3. Shakoor, R., Hassan, M. Y., Raheem, A., \& Wu, Y. K.: Wake effect modeling: A review of wind farm layout optimisation using Jensen' s model. Renewable and Sustainable Energy Re-views, 58 (2016) 1048-1059.

4. Funke, S. W., Farrell, P. E., Piggott, M. D.: Tidal turbine array optimisation using the adjoint approach. Renewable Energy, 63 (2014) 658-673.

5. Du Feu, R. J., Funke, S. W., Kramer, S. C., Culley, D. M., Hill, J., Halpern, B. S., \& Piggott, M. D.: The trade-off between tidal-turbine array yield and impact on flow: A multi-objective optimisation problem. Renewable Energy, 114 (2017) 1247-1257.

6. González-Gorbeña, E., Qassim, R. Y., Rosman, P. C.: Optimisation of hydrokinetic turbine array layouts via surrogate modelling. Renewable Energy, 93 (2016) 45-57.

7. Queipo, N. V., Haftka, R. T., Shyy, W., Goel, T., Vaidyanathan, R., \& Tucker, P. K.. Surrogate-based analysis and optimisation. Progress in aerospace sciences, 41 (1) (2005) 1-28.

8. González-Gorbeña, E., Qassim, R. Y., \& Rosman, P. C.: Multi-dimensional optimisation of Tidal Energy Converters array layouts considering geometric, economic and environmental constraints. Renewable energy, 116 (2018) 647-658.

9. González-Gorbeña, E., Pacheco, A., Plomaritis, T. A., Ferreira, Ó., Sequeira, C.: Estimating the optimum size of a tidal array at a multi-inlet system considering environmental and perfor-mance constraints. Applied Energy, 232 (2018) 292-311.

10. Pacheco A, Vila-Concejo A, Ferreira Ó, Dias J.A.: Assessment of tidal inlet evolution and stability using sediment budget computations and hydraulic parameter analysis. Marine Geol-ogy, 247 (2008) 104-27.

11. Koziel, S., Tesfahunegn, Y., Leifsson, L.: Variable-fidelity CFD models and co-Kriging for expedited multi-objective aerodynamic design optimisation. Engineering Computations, 33 (8) (2016) 2320-2338.

12. Acar, E., Tüten, N., Güler, M.A.: Design optimisation of an automobile torque arm using global and successive surrogate modeling approaches. Proc. Inst. Mech. Eng. Part D: J. Automob. Eng. 233 (6) (2018) 1453-1465.

13. Koziel, S., Ogurtsov, S.: Surrogate-based optimisation. In: Antenna Design by SimulationDriven Optimisation, Springer, Cham, (2014) 13-24.

14. Sacks, J., Welch, W. J., Mitchell, T. J., Wynn, H. P.: Design and analysis of computer experiments. Statistical science, (1989) 409-423.

15. Loeppky, J. L., Sacks, J., Welch, W. J.: Choosing the sample size of a computer experiment: A practical guide. Technometrics 51(4) (2009) 366-376.

16. Eisenmann, E. G. G., Qassim, R. Y., \& Rosman, P. C. C.: A metamodel simulation based optimisation approach for the tidal turbine location problem. Aquatic Science and Technology 3(1) (2015), 33-58.

17. Delft3D Open Source Community. Available at: https://oss.deltares.nl/web/delft3d/home

18. Kai-Tai, F., Runze, L., Agus, S.. Design and modeling for computer experiments. Computer Science \& Data Analysis Series. Ed. Chapman \& Hall/CRC (2006).

19. Buhmann, M. D.: Radial basis functions: theory and implementations. Cambridge university press, (2003).

20. Forrester, A., Sobester, A., Keane, A.: Engineering design via surrogate modelling: a practical guide. John Wiley \& Sons, (2008). 